\title{
REFERENCES
}

1. Jacques Herbrand, Recherches sur la theorie de la demonstration, Travaux de la Société des Sciences et des Lettres de Varsovie, Classe III sciences mathématiques et physiques, no. 33 (1930).

2. - Sur le probleme fondamental de la logique mathematique, Comptes Rendus des séances de la Société des Sciences et des Lettres de Varsovie, Classe III sciences mathématiques et physiques, no. 24 (1931).

3. John van Heijenoort, Editor, Jacques Herbrand, Ecrits Logiques, Presses Universitaires, Paris (to appear)

HARVARD UNIVERSITY AND

PrINCETON UNIVERSITY

\section{SIMPLY INVARIANT SUBSPACES 1}

BY T. P. SRINIVASAN

Communicated by Edwin Hewitt, March 18, 1963

Let $L^{1}, L^{2}$ denote respectively the spaces of summable and square summable functions on the circle group and $H^{1}, H^{2}$ their subspaces consisting of those functions whose Fourier coefficients vanish for negative indices. A closed subspace $M$ of $L^{1}$ or $L^{2}$ is "invariant" if

$$
x M \subset M
$$

and "simply invariant" if the above inclusion is strict, where $\chi$ is the character

$$
\chi(x)=e^{i x} .
$$

The structure of simply invariant subspaces is known, namely, they are precisely the subspaces of the form $q H^{1}$ or $q H^{2}$ (respectively) where $q$ is a measurable function of modulus 1 a.e. Beurling [1] first proved this for subspaces $M \subset H^{2}$; for $M \subset H^{1}$, this is due to de LeeuwRudin [5]; for $M \subset L^{2}$, due to Helson-Lowdenslager [3] and for $M \subset L^{1}$, due to Forelli [2]. In [3] Helson-Lowdenslager also gave a simple proof of the $H^{2}$ case, free of function theoretic considerations. Using their arguments Hoffman [4] extended this result to simply invariant subspaces of $\boldsymbol{H}^{2}(\mathrm{dm})$ defined over logmodular algebras. In this paper we prove this result for simply invariant subspaces of $L^{2}(d m)$ and $L^{1}(d m)$ over logmodular algebras; the results of the previous authors follow as a corollary. The proofs of the previous authors

\footnotetext{
1 This work was done while I held a visiting appointment at the University of California, Berkeley.

I thank Professors Helson and Ju-kwei Wang for the useful discussions I had with them.
} 
do not extend to this general case as they depend on facts which either have no analogues or are not true for the logmodular algebras; when specialised to their contexts, our proof turns out to be even simpler. Our proof for the case of $L^{2}(d m)$ was inspired by that of HelsonLowdenslager for the $H^{2}$ case and is in the same spirit as theirs.

Let $X$ be a compact Hausdorff space and $\boldsymbol{A}$ a subalgebra of the algebra $C(X)$ of complex continuous functions on $X$ with the uniform norm.

\section{$\boldsymbol{A}$ is logmodular if}

i. $A$ is uniformly closed,

ii. $A$ contains the constant functions,

iii. $\boldsymbol{A}$ separates the points of, $X$ and

iv. the set of functions $\log |f|$ where $f, 1 / f \in A$, is uniformly dense in the algebra of real continuous functions on $X$.

Let $m$ be a probability Baire measure on $X$ which is "multiplicative" on $A$, meaning

$$
\int f g d m=\int f d m \int g d m
$$

for all $f, g \in A$ (such measures always exist), and let $\boldsymbol{H}^{1}(d m), \boldsymbol{H}^{2}(d m)$ denote the closures of $\boldsymbol{A}$ in $L^{1}(d m), L^{2}(d m)$ respectively. The invariant subspaces $M$ are now closed subspaces of $L^{1}(d m), L^{2}(d m)$, which are invariant under multiplication by functions in $\boldsymbol{A}$ or equivalently by functions in $\boldsymbol{A}_{0}$, where

$$
\boldsymbol{A}_{0}=\left\{f \mid f \in A, \int f d m=0\right\}
$$

and the simply invariant $\boldsymbol{M}$ 's are those for which the inclusion $A_{0} M \subset M$ is strict. ${ }^{2}$

In the case considered earlier, $X$ was the unit circle, $A_{0}$ was the uniform closure of the algebra generated by $\chi$ in $C(X)$ and $m$ the normalised Lebesgue measure. We have

THEOREM.

1. The simply invariant subspaces of $L^{2}(d m)$ are precisely the subspaces of the form $q H^{2}(d m)$ where $q \in L^{2}(d m)$ and $|q|=1$ a.e. $(d m)$.

2. The simply invariant subspaces of $L^{1}(d m)$ are precisely the subspaces of the form $q H^{1}(d m)$ where $q \in L^{1}(d m)$ and $|q|=1$ a.e. $(d m) .^{8}$

? $A_{0} M$ should be replaced by its closure in $L^{2}(d m)$ respectively $L^{1}(d m)$, which necessitates changes in the proof.

3 The details of the proof of the $L^{1}$ theorem and its function theoretic consequences will be published separately. 
PRoof. It is obvious that subspaces of the form $q H^{2}(d m), q H^{1}(d m)$ are invariant; they are simply invariant because for instance, $q \in q \boldsymbol{H}^{2}(d m), q \boldsymbol{H}^{1}(d m)$ while $q \notin q \boldsymbol{A}_{0} \boldsymbol{H}^{2}(d m), q \boldsymbol{A}_{0} \boldsymbol{H}^{1}(d m)$. To prove the converse:

1. We need the following facts about logmodular algebras [4, pp. 284, 293]:

(a) $A+\bar{A}$ is dense in $L^{2}(d m)$ where the bar denotes complex conjugation,

(b) if $\mu$ is any positive Baire measure on $X$ such that $\int f d \mu=0$ for all $f \in \boldsymbol{A}_{0}$ then $d \mu=c d m$ for some constant $c$.

Now let $M \subset L^{2}(d m)$ be simply invariant and let $q \in M \ominus \boldsymbol{A}_{0} \boldsymbol{M}$, $q \neq 0$. Then $q \perp \boldsymbol{A}_{0} q$, so $\int f|q|^{2} d m=0$ for all $f \in \boldsymbol{A}_{0}$ and by (b), $|q|^{2}$ $=c$ a.e. By modifying $q$ we may assume that $|q|=1$ a.e.

Clearly $q H^{2}(d m) \subset M$, because of invariance of $M$. Let $g \in M \ominus q H^{2}(d m)$. Then $g \perp q \boldsymbol{A}$, so $g \bar{q} \perp \boldsymbol{A}$. Also $\boldsymbol{A}_{0} g \subset \boldsymbol{A}_{0} \boldsymbol{M}$, so $q \perp \boldsymbol{A}_{0} g$ so that $g \bar{q} \perp \overline{\boldsymbol{A}}_{0}$. Thus $g \bar{q} \perp \boldsymbol{A}+\overline{\boldsymbol{A}}$, hence $g \bar{q}=0$ a.e. by (a) and since $|q|=1$ a.e., $g=0$. Thus $\boldsymbol{M}=q \boldsymbol{H}^{2}(d m)$.

2. We use (1) to prove (2). Let $N \subset L^{1}(d m)$ be simply invariant and let $M=N \cap L^{2}(d m) . M$ is clearly an invariant subspace of $L^{2}(d m)$. We shall show that it is actually simply invariant. Let $f \in N$. We can find $f_{1}, f_{2} \in L^{2}(d m)$ such that $f=f_{1} f_{2}$; we may also assume that one of them, say, $f_{2}$ is nonzero a.e. Then $f_{2} H^{2}(d m)$ is a simply invariant subspace of $L^{2}(d m)$ and is by (1) of the form $q_{2} H^{2}(d m),\left|q_{2}\right|=1$ a.e. Now

$$
f_{1} q_{2} \in f_{1} q_{2} H^{2}(d m)=f_{1} f_{2} H^{2}(d m)=f H^{2}(d m) \subset N .
$$

Also $f_{1} q_{2} \in L^{2}(d m)$. Hence $f_{1} q_{2} \in M$. Suppose $\boldsymbol{M}=\boldsymbol{A}_{0} \boldsymbol{M}$. Then $f_{1} q_{2}$ $\in A_{0} M$. Let

$$
f_{1} q_{2}=f_{0} g, \quad f_{0} \in A_{0}, \quad g \in M \subset N
$$

and

$$
f_{2}=q_{2} h, \quad h \in H^{2}(d m) .
$$

Then

$$
f=f_{1} f_{2}=f_{1} q_{2} h=f_{0} g h \in A_{0} N H^{2}(d m) \subset A_{0} N
$$

and it follows that $N=A_{0} N$. Hence if $N$ is simply invariant, so is $M$.

Let then $M=q H^{2}(d m)$ by (1). We shall show that $\boldsymbol{N}=q \boldsymbol{H}^{1}(d m)$. Clearly $q H^{1}(d m) \subset N$. Let $f \in N$ and $f_{1}, f_{2}, q_{2}, h$ be as above. Then $f_{1} q_{2} \in M=q H^{2}(d m)$. Let $f_{1} q_{2}=g h^{\prime}, h^{\prime} \in H^{2}(d m)$. Then

$$
f=f_{1} f_{2}=f_{1} q_{2} h=q h^{\prime} h \in q H^{1}(d m)
$$

as $h^{\prime}, h \in H^{2}(d m)$. It follows that $N=q H^{1}(d m)$. 
We may remark that if $M \subset H^{2}(d m)$ is invariant and we assume with Hoffman [4, p. 293] that $\int g d m \neq 0$ for at least one $g \in M$ then $M$ is certainly simply invariant and Hoffman's result follows. But this latter condition is not necessary for simple invariance as the example of $z^{k} H^{2}, k \geqq 1$ shows.

\section{REFERENCES}

1. A. Beurling, On two problems concerning linear transformations in Hilbert space, Acta Math. 81 (1949), 239-255.

2. F. Forelli, Invariant subspaces in L1, Proc. Amer. Math. Soc. 14 (1963), 76-79.

3. H. Helson and D. Lowdenslager, Invariant subspaces, Proc. Internat. Sympos. on Linear Spaces, Jerusalem, 1960, pp. 251-262.

4. K. Hoffman, Analytic functions and logmodular Banach algebras, Acta Math. 108 (1962), 271-317.

5. K. de Leeuw and W. Rudin, Extreme points and extremum problems in $H_{1}$, Pacific J. Math. 8 (1958), 467-485.

Panjab University, Chandigarh, India and

University of California, Berkeley 